\title{
Comparative analysis of single nucleotide polymorphisms in the nuclear, chloroplast, and mitochondrial genomes in identification of phylogenetic association among seven melon (Cucumis melo L.) cultivars
}

\author{
Qianglong Zhu ${ }^{1,2)}$, Peng Gao ${ }^{1,2)}$, Shi Liu ${ }^{1,2)}$, Sikandar Amanullah ${ }^{1,2)}$ and Feishi Luan*1,2) \\ 1) Horticulture College, Northeast Agricultural University, Harbin, Heilongjiang 150030, China \\ 2) Key Laboratory of Biology and Genetic Improvement of Horticulture Crops (Northeast Region), Ministry of Agriculture, Harbin, \\ Heilongjiang 150030, China
}

\begin{abstract}
A variety of melons are cultivated worldwide, and their specific biological properties make them an attractive model for molecular studies. This study aimed to investigate the single nucleotide polymorphisms (SNPs) from the mitochondrial, chloroplast, and nuclear genomes of seven melon accessions (Cucumis melo L.) to identify the phylogenetic relationships among melon cultivars with the Illumina HiSeq 2000 platform and bioinformatical analyses. The data showed that there were a total of 658 mitochondrial SNPs (207-295 in each), while there were 0-60 chloroplast SNPs among these seven melon cultivars, compared to the reference genome. Bioinformatical analysis showed that the mitochondrial tree topology was unable to separate the melon features, whereas the maximum parsimony/neighbor joining (MP/NJ) tree of the chloroplast SNPs could define melon features such as seed length, width, thickness, 100-seed weight, and type. SNPs of the nuclear genome were better than the mitochondrial and chloroplast SNPs in the identification of melon features. The data demonstrated the usefulness of mitochondrial, chloroplast, and nuclear SNPs in identification of phylogenetic associations among these seven melon cultivars.
\end{abstract}

Key Words: melon, SNPs, chloroplast genome, mitochondrial genome, nuclear genome, bioinformatical analysis.

\section{Introduction}

Melon (Cucumis melo L.) is one of the most widely cultivated horticultural crops in the world. Melon first originated in Africa and Southwest Asia (Vaughan and Geissler 2009) and then spread worldwide. In 2013, the global melon production was more than 29 million tons (http://faostat.fao. org). Melon is an attractive model for molecular studies owing to its specific biological properties (Garcia-Mas et al. 2012) such as fruit ripening (Pech et al. 2008, Saladié et al. 2015), sex determination (Boualem et al. 2008, Martin et al. 2009), and carotenoid content (Harel-Beja et al. 2010, Ibdah et al. 2006, Tzuri et al. 2015). The economic importance and research value of melons would promote the completion of the genome sequence of its mitochondria, chloroplast, and nucleus (Garcia-Mas et al. 2012, Rodriguez-Moreno et al. 2011). Both chloroplast and nuclear genomes are often

Communicated by Sachiko Isobe

Received April 1, 2016. Accepted August 17, 2016.

First Published Online in J-STAGE on October 18, 2016.

*Corresponding author (e-mail: luanfeishi@neau.edu.cn) used for molecular phylogenies to identify the diversification and evolutionary history of cultivated melons (Chung et al. 2006, Renner et al. 2007, Sebastian et al. 2010, Tanaka et al. 2013). However, the mitochondrial genome has been underutilized as a source of phylogenetic data on melons, which may be attributed to a generally low rate of nucleotide substitution due to the impact of RNA editing (Govindarajulu et al. 2015), coupled with highly divergent noncoding regions and ubiquitous rearrangements (Rodriguez-Moreno et al. 2011), which could impact its usage for phylogenetic reconstruction (Govindarajulu et al. 2015, Straub et al. 2012). Accumulative evidence supports the analysis of the mitochondrial genome for phylogenetic reconstruction because of the fact that the mitochondrial genome is paternally inherited and transmitted through pollen, like other species of Cucumis (Havey 1997, Havey et al. 1998, Shen et al. 2013, Wei et al. 2011, Zhao et al. 2014). The mitochondrial genome of melon contains more than $2,740 \mathrm{~kb}$ of nucleotides, the largest one among the plants of Cucurbitaceae family. In contrast, the chloroplast is maternally inherited, although its genome contains only $156,017 \mathrm{bp}$ of nuclear acids. A comprehensive analysis of their genomes 
could provide a complementary rather than redundant insight into the reticulate evolutionary history of melons.

During the past decades, there have been numerous attempts to identify and evaluate the chloroplast and nuclear genomes as molecular markers to characterize melon biodiversity and to explain phylogenetic relationships using techniques such as random-amplified polymorphic DNA (RAPD) (Nakata et al. 2005, Tanaka et al. 2007), simple sequence repeats (SSR) (Chung et al. 2006, Gao et al. 2012, Nakata et al. 2005), amplified fragment length polymorphism (AFLP) (Garcia-Mas et al. 2000), and single nucleotide polymorphism (SNP) (Sanseverino et al. 2015, Tanaka et al. 2013). Recently, various studies utilized nearly complete chloroplast and nuclear genome approach to identify molecular biomarkers for phylogenetic analysis (Govindarajulu et al. 2015, Sabir et al. 2014, Straub et al. 2012) because of a significant decrease in the financial cost for DNA sequencing and the next generation sequencing (NGS) technology. Assessment of genomic SNP is more popular and frequently used among molecular markers for genomic studies (Govindarajulu et al. 2015, Sabir et al. 2014, Straub et al. 2012, Zhang et al. 2012). Straub et al. (2012) and Govindarajulu et al. (2015) implemented a genome-skimming approach to assemble the mitochondrial and chloroplast genomes based on reference-guided assembly to find the polymorphic sites of both genomes. Zhang et al. (2012) mapped mate-paired reads to two organellar genomes to identify intra-specific SNPs. Sabir et al. (2014) filtered the reads mapped to both the mitochondrial and chloroplast genomes, and the remaining reads were used for all subsequent genome SNP analysis.

In this study, we sequenced the mitochondrial, chloroplast, and nuclear genomes of seven melon cultivars from different countries and identified all SNPs in order to investigate their genetic diversity and phylogenetic relationships. This study expects to evaluate the effect of DNA transfer among these three genomes on organellar SNP analysis and to investigate whether genetic polymorphism exits in the mitochondrial and chloroplast genomes of melon cultivars. In addition, the study aims to investigate whether organellar SNPs are useful to separate melon cultivars and whether there is a difference in the mitochondrial, chloroplast, and nuclear genomes as molecular biomarkers to identify the phylogenetic relationships. Overall, we expected to utilize these SNPs to identify their organellar phylogenies and to construct the nuclear phylogeny using these SNP markers from the nuclear genome.

\section{Materials and Methods}

\section{Sample collection and DNA extraction}

Seeds of the seven melon accessions were kindly provided by the College of Horticulture (Northeast Agricultural University, Harbin, China) and selected as representatives of the main groups (Table 1, Fig. 1). Some of these accessions were parental lines for several melon mapping populations, which have been extensively used for constructing genetic maps and mapping agronomical important traits. The genotype of three published melon reference genomes (Garcia-Mas et al. 2012, Rodriguez-Moreno et al. 2011) was also included as a control in our analysis.

About $500 \mathrm{mg}$ of field-collected leaf tissues of each of these seven cultivars were collected and snap-frozen in liquid nitrogen. Genomic DNA was extracted using a modified DNA extraction method, according to a previous study (Allen et al. 2006). To remove RNA contamination, the samples were digested with $10 \mathrm{mg} / \mathrm{mL}$ of RNase A (Sigma, ST Louis, MO, USA) at $37^{\circ} \mathrm{C}$ for $30 \mathrm{~min}$. DNA concentration was measured by an SMA3000 spectrophotometer (Plextech, Shenzhen, China) with an optical density at $260 \mathrm{~nm}$, and these DNA samples were sequenced by Beijing Genomics Institute (BGI; Shenzhen, China) with the Illumina HiSeq 2000 platform.

\section{Genome sequencing, reads mapping, and SNP analysis}

Each DNA sample had approximately 60 million 90-bp paired-end reads, with an average of $19 \times$ depths. Seven DNA sequencing libraries with 500-bp fragment length were generated and the raw data were processed in two steps, i.e., adapter sequences in the reads were trimmed and the reads with more than $30 \%$ low quality bases (quality value $\leq 5$ ) were removed. For SNP analysis of the mitochondrial and chloroplast genomes, the remaining DNA sequencing reads from these seven samples were randomly drawn for $0.5 \times$ dataset covered of nuclear genome for three times as triplicate repeat subsamples using SEQTK (https://

Table 1. Melon cultivars used in the study

\begin{tabular}{|c|c|c|c|c|c|c|}
\hline Cultivar & Geographic location & Fruit shape & Fruit skin color & $\begin{array}{l}\text { Seed length/width/ } \\
\text { thickness }(\mathrm{mm})\end{array}$ & 100-seed weight (g) & Seed type ${ }^{a}$ \\
\hline Topmark & America & Cylindrical & Green & $11.0 / 4.9 / 1.5$ & 3.7 & $\mathrm{~L}$ \\
\hline MR-1 & America & Oval & Orange & $10.3 / 4.9 / 2.2$ & 2.5 & $\mathrm{~L}$ \\
\hline M4-7 & China & Round & White & $10.6 / 4.8 / 1.8$ & 3.8 & $\mathrm{~L}$ \\
\hline M4-5 & China & Round & Yellow & $9.3 / 4.0 / 2.1$ & 2.5 & $\mathrm{~L}$ \\
\hline M1-15 & China & Cylindrical & White & $6.2 / 3.0 / 1.3$ & 1.2 & $\mathrm{~S}$ \\
\hline M1-7 & China & Cylindrical & White & $6.0 / 3.2 / 1.1$ & 0.8 & $\mathrm{~S}$ \\
\hline M1-33 & China & Cylindrical & Green & $6.8 / 3.3 / 1.4$ & 1.5 & $\mathrm{~S}$ \\
\hline DHL92 & Spain & - & - & - & - & - \\
\hline
\end{tabular}

${ }^{a}$ Based on the seed length and 100-seed weight, melon is classified into large-seed type (L) ( $\geq 9.0 \mathrm{~mm}$ or $\geq 2.5 \mathrm{~g}$ ) and small-seed type (S) $(<9.0 \mathrm{~mm}$ or $<2.5 \mathrm{~g})$ according to the criteria reported by Tanaka et al. (2013). 

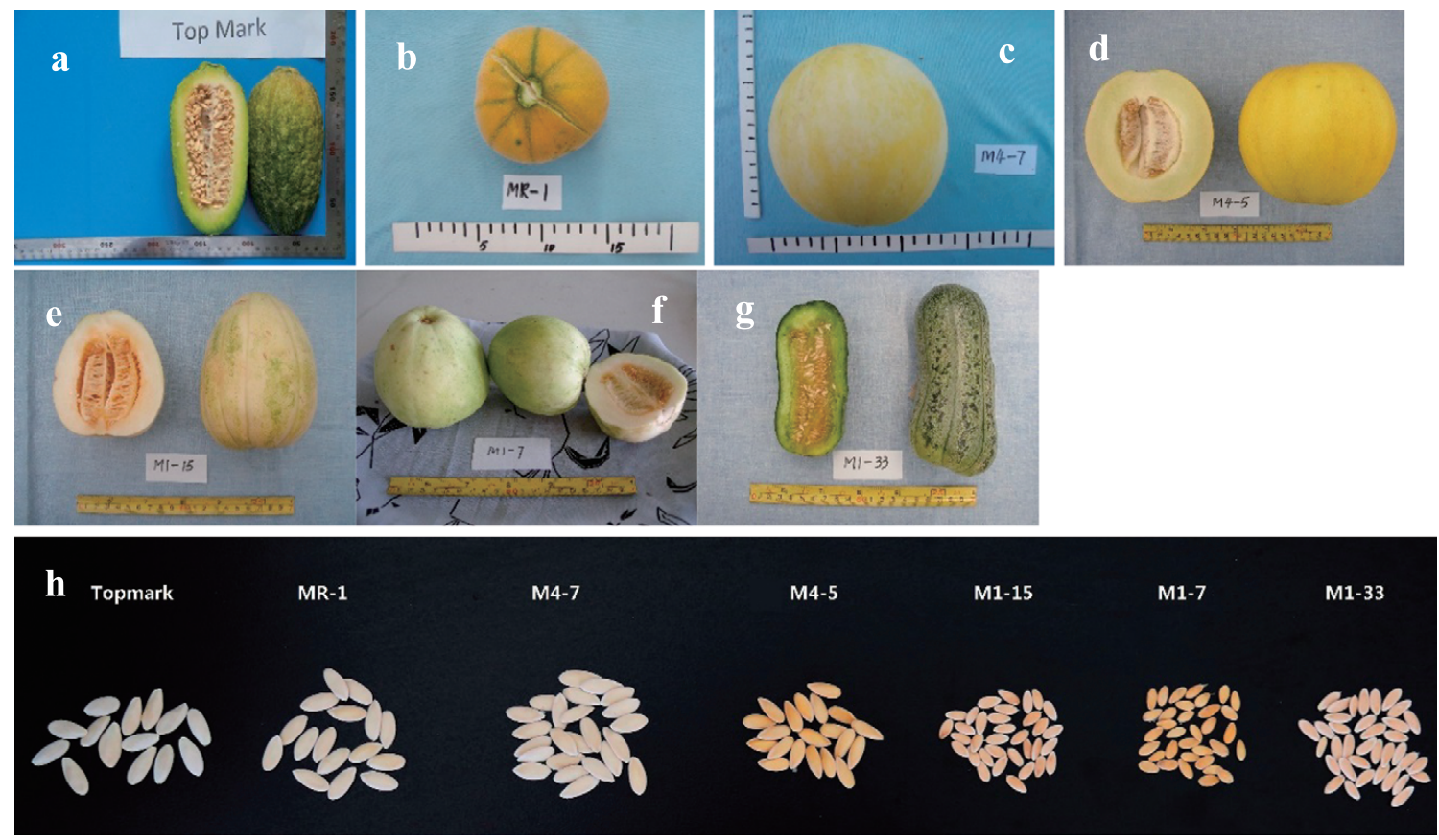

Fig. 1. Fruits from Topmark (a), MR-1 (b), M4-7 (c), M4-5 (d), M1-7 (e), M1-15 (f) and M1-33 (g). Seeds from mature fruits of the seven melon cultivars (h).

github.com/lh3/seqtk). Individual read pools of each melon accession were aligned separately to the melon mitochondrial (JF412792 to JF412800) and chloroplast (NC_015983) reference genomes using BWA v0.7.12-r1039 (Li 2013). All reads that aligned to both chloroplast and mitochondrial genomes were removed. Only filtered reads were further run through SAMTOOLS v1.2 mpileup (Li et al. 2009) and VARSCAN v2 (Koboldt et al. 2012) pipelines to identify SNPs unique to the mitochondrial and chloroplast genomes, respectively. Homogeneous SNPs with a read depth $\geq 10$, mapping quality $\geq 20$, and SNP quality $\geq 15$ were retained and heterogeneous SNPs were filtered. The SNPs of intersection among the three repeat subsamples were used to predict the effects of the variants on genes through SnpEFF v4.2 (Cingolani et al. 2012). For SNP analysis of melon nuclear genome, all remaining clean reads of these seven melon varieties after quality control were directly used to identify SNPs according to the above methods. About 50,000 homozygous SNPs of the nuclear genome were then randomly extracted from the results of SNP identification and used to determine the phylogenetic association among these seven melon cultivars.

\section{Alignment and phylogenetic analyses}

The sequence matrix of SNPs from eight nuclear, mitochondrial, and chloroplast genomes (seven from this study and DHL92 from MELONOMICS, Table 1) was aligned using CLUSTALX v2.0.12 (Larkin et al. 2007). The phylogenetic relationship was determined using the neighbor joining (NJ) method with a bootstrapping of 1,000 replications with the MEGA v6 (Tamura et al. 2013). The phylo- genetic relationship was also analyzed using the maximum parsimony (MP) method with PAUP* v4.0a147 (Swofford 2002). The phylogenies constructed from the mitochondrial, chloroplast, and nuclear genomes were visually inspected to identify the incongruence in genus relationships.

\section{Results}

Mapping of DNA sequence reads to the reference genomes

The number of DNA sequence reads from each sample ranged 58.92-66.60 million; $91.89 \%$ of these clean reads were mapped to the nuclear genome with an average coverage of $80.27 \%$ (Table 2 ), while $2,222,222$ or $0.5 \times$ reads covering the melon nuclear genome were randomly extracted from the total reads of every sample as triplicate repeat subsamples. Mapping of the $0.5 \times$ reads to the mitochondrial (JF412792 to JF412800) and chloroplast genome (NC_015983) was performed. The average number of the reads mapped to the mitochondrial genome varied between 501,439 and 693,487 , depending on the cultivar and leading to $22.56-31.21 \%$ of the $0.5 \times$ reads, while $221,468-459,552$ reads, representing $9.97-20.68 \%$ of the $0.5 \times$ reads, mapped to the chloroplast genome of the seven cultivars (Table 3 ). Since $1.41 \%$ or $38.6 \mathrm{~kb}$ of the $2.74-\mathrm{Mb}$ mitochondrial genome represented chloroplast insertions (Rodriguez-Moreno et al. 2011), the reads that mapped to both genomes were removed and the remaining reads were mapped to the reference chloroplast and mitochondrial genomes to avoid generating false SNPs due to DNA sequences that were transferred from the chloroplast to the mitochondrial genomes. Mapping of the remaining reads after filtering by both the 
Table 2. Alignment of DNA sequencing reads to the nuclear reference genome (Melon_genome_v3.5.1)

\begin{tabular}{lcccc}
\hline \hline Cultivar & Total reads & $\begin{array}{c}\text { Number of } \\
\text { reads mapped }\end{array}$ & Depth & Coverage \\
\hline Topmark & $58,916,472$ & $\begin{array}{c}54,939,610 \\
(93.25 \%)\end{array}$ & 19 & $79.93 \%$ \\
MR-1 & $66,600,000$ & $\begin{array}{c}60,965,640 \\
(91.54 \%)\end{array}$ & 19 & $79.99 \%$ \\
M4-7 & $60,514,798$ & $\begin{array}{c}54,463,318 \\
(90.00 \%)\end{array}$ & 18 & $80.01 \%$ \\
M4-5 & $60,196,095$ & $\begin{array}{c}54,477,466 \\
(90.50 \%)\end{array}$ & 19 & $79.96 \%$ \\
M1-15 & $61,230,665$ & $\begin{array}{c}56,687,350 \\
(92.58 \%)\end{array}$ & 19 & $80.95 \%$ \\
M1-7 & $60,142,435$ & $\begin{array}{c}55,752,037 \\
(92.70 \%)\end{array}$ & 20 & $80.98 \%$ \\
M1-33 & $60,316,345$ & $55,883,094$ \\
& & 21 & $80.12 \%$ \\
Average & $61,130,972$ & $\begin{array}{c}(96,166,930 \\
(91.89 \%)\end{array}$ & 19 & $80.27 \%$ \\
& & & \\
\hline
\end{tabular}

reference genomes resulted in $93.10-96.83 \%$ and 64.15 $64.41 \%$ breadth coverage with the average depth of $21-$ $32 \mathrm{bp}$ and $190-400 \mathrm{bp}$ of the mitochondrial and chloroplast genome, respectively. Thus, in the mitochondrial genome, this reduced the number of mapped reads to 324,316 496,945 for the seven cultivars, which represented 14.59 $22.36 \%$ of the $0.5 \times$ reads before filtering (Table 3 ). In the case of the chloroplast genome, the number of reads mapped was reduced to $116,149-250,503$ or $5.23-11.27 \%$ of the $0.5 \times$ reads before filtering (Table 4 ). Filtering out reads that mapped to both genomes reduced the number of SNPs detected in both the mitochondrial and chloroplast genomes and also slightly reduced the read depth and coverage, but was enough for the identification of SNPs.

\section{Identification of mitochondrial SNPs}

The number of mitochondrial SNPs in each of these seven melon cultivars compared to the reference genome ranged 207-295, with a total of 658 SNPs (Fig. 2A), while 182 of the 658 SNPs were unique to a single cultivar and resulted from only three melon cultivars (Topmark, M4-5 and M1-15). A high SNP occurred between large-seed and small-seed melon groups, and the large-seed melons had more polymorphisms than the small-seed ones. The 658 SNPs were localized at the different loci of the mitochondrial genome and a majority of the SNPs were localized in the intergenic spacer regions and intron; only two nonsynonymous substitutions (SJF412792 222328 and SJF412792 222329) and two synonymous substitutions (SJF412792 2137706 and SJF412792 2138399) were localized in the rps1 and atpl gene, respectively (Fig. 3, Supplemental Table 1).

\section{Identification of chloroplast SNPS}

The number of chloroplast SNPs in the melon cultivars ranged 0-60; all chloroplast SNPs were from large-seed melons while none was observed among the small-seed melons or the reference chloroplast genome (Fig. 2B). However, there were only two SNP markers (SNC_015983 62605 and SNC_015983_85814) unique in Topmark and M4-5, respectively (Supplemental Table 2, Fig. 2B). Although the number of chloroplast SNPs was much smaller than that of the mitochondrial genome, a high genetic polymorphism was detected between small-seed and large-seed melons. These 63 chloroplast SNPs were localized in different loci of the chloroplast genome and nearly half of them were in exons, 0 in introns, and 37 in intergenic spacers. With regard to the genic SNPs, 18 of them resulted in nonsynonymous changes and eight were synonymous substitutions (Fig. 3, Supplemental Table 2).

Table 3. Alignment of DNA sequencing reads to the mitochondrial reference genome (JF412792 to JF412800)

\begin{tabular}{|c|c|c|c|c|c|}
\hline Cultivar & Total reads & Number of $0.5 \times$ reads mapped & $\begin{array}{c}\text { Number of } 0.5 \times \text { reads mapped after } \\
\text { filtering }\end{array}$ & $\begin{array}{l}\text { Depth after } \\
\text { filtering }\end{array}$ & $\begin{array}{c}\text { Coverage after } \\
\text { filtering }\end{array}$ \\
\hline Topmark & $58,916,472$ & $501,439 \pm 366(22.56 \pm 0.02 \%)$ & $363,705 \pm 243(16.37 \pm 0.01 \%)$ & 23 & $96.10 \pm 0.01 \%$ \\
\hline MR-1 & $66,600,000$ & $523,768 \pm 251 \quad(23.57 \pm 0.01 \%)$ & $339,308 \pm 542(15.27 \pm 0.02 \%)$ & 22 & $96.12 \pm 0.01 \%$ \\
\hline M4-7 & $60,514,798$ & $693,487 \pm 1111(31.21 \pm 0.05 \%)$ & $496,233 \pm 891(22.33 \pm 0.04 \%)$ & 32 & $96.81 \pm 0.07 \%$ \\
\hline M4-5 & $60,196,095$ & $629,989 \pm 742 \quad(28.35 \pm 0.03 \%)$ & $496,945 \pm 445(22.36 \pm 0.02 \%)$ & 32 & $96.83 \pm 0.02 \%$ \\
\hline M1-15 & $61,230,665$ & $533,365 \pm 801 \quad(24.00 \pm 0.04 \%)$ & $324,316 \pm 620(14.59 \pm 0.03 \%)$ & 21 & $93.10 \pm 0.04 \%$ \\
\hline M1-7 & $60,142,435$ & $523,716 \pm 501 \quad(23.57 \pm 0.02 \%)$ & $409,483 \pm 505(18.43 \pm 0.02 \%)$ & 27 & $95.31 \pm 0.02 \%$ \\
\hline M1-33 & $60,316,345$ & $537,883 \pm 458 \quad(24.20 \pm 0.02 \%)$ & $435,170 \pm 389(19.58 \pm 0.02 \%)$ & 29 & $95.10 \pm 0.01 \%$ \\
\hline
\end{tabular}

Table 4. Alignment of DNA sequencing reads to the chloroplast reference genome (NC_015983)

\begin{tabular}{|c|c|c|c|c|c|}
\hline Cultivar & Total reads & Number of $0.5 \times$ reads mapped & $\begin{array}{c}\text { Number of } 0.5 \times \text { reads mapped after } \\
\text { filtering }\end{array}$ & $\begin{array}{l}\text { Depth after } \\
\text { filtering }\end{array}$ & $\begin{array}{c}\text { Coverage after } \\
\text { filtering }\end{array}$ \\
\hline Topmark & $58,916,472$ & $302,422 \pm 405(13.61 \pm 0.02 \%)$ & $164,687 \pm 110 \quad(7.41 \pm 0.01 \%)$ & 260 & $64.30 \pm 0.02 \%$ \\
\hline MR-1 & $66,600,000$ & $405,942 \pm 484(18.27 \pm 0.02 \%)$ & $221,481 \pm 542 \quad(9.97 \pm 0.02 \%)$ & 370 & $64.25 \pm 0.10 \%$ \\
\hline M4-7 & $60,514,798$ & $396,608 \pm 471(17.85 \pm 0.02 \%)$ & $119,353 \pm 644 \quad(8.97 \pm 0.03 \%)$ & 320 & $64.16 \pm 0.04 \%$ \\
\hline M4-5 & $60,196,095$ & $267,155 \pm 362(12.02 \pm 0.02 \%)$ & $134,110 \pm 304 \quad(6.03 \pm 0.01 \%)$ & 220 & $64.19 \pm 0.10 \%$ \\
\hline M1-15 & $61,230,665$ & $459,552 \pm 409(20.68 \pm 0.02 \%)$ & $250,503 \pm 227(11.27 \pm 0.01 \%)$ & 400 & $64.41 \pm 0.10 \%$ \\
\hline M1-7 & $60,142,435$ & $230,382 \pm 196(10.37 \pm 0.01 \%)$ & $116,149 \pm 175 \quad(5.23 \pm 0.01 \%)$ & 190 & $64.15 \pm 0.04 \%$ \\
\hline M1-33 & $60,316,345$ & $221,468 \pm 246 \quad(9.97 \pm 0.01 \%)$ & $118,755 \pm 395 \quad(5.34 \pm 0.02 \%)$ & 200 & $64.16 \pm 0.06 \%$ \\
\hline
\end{tabular}




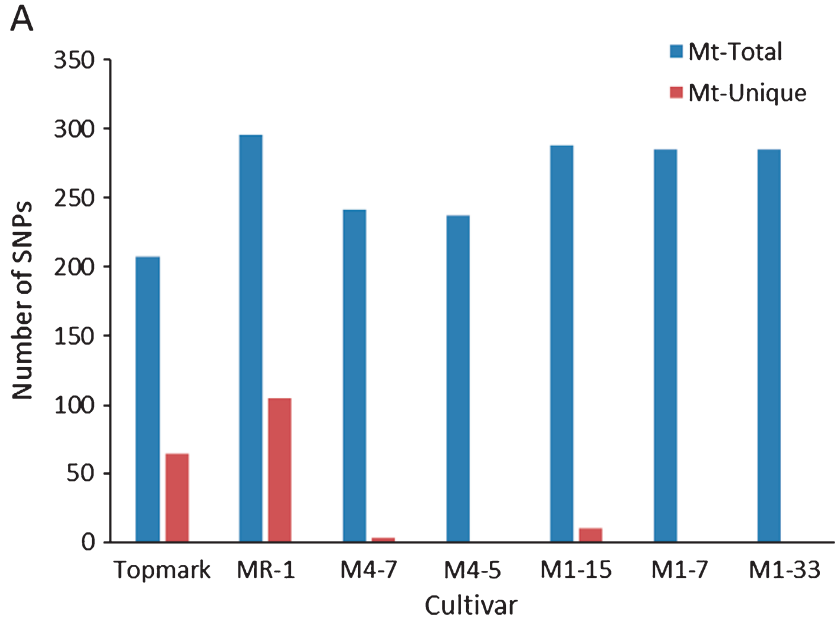

B

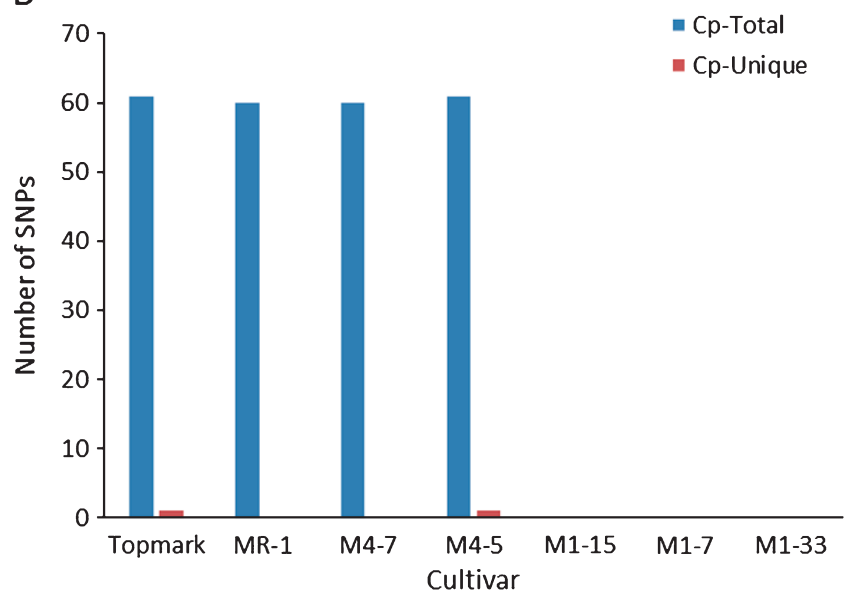

Fig. 2. Identification of single nucleotide polymorphisms (SNPs) from the mitochondrial and chloroplast genomes. Number of total and unique SNPs detected from each of the seven melon cultivars. (A) Mitochondria (Mt), (B) Chloroplast (Cp).

Mitochondrial and chloroplast SNPS to distinguish between the features of the melon cultivars

Unrooted maximum parsimony (MP) trees and neighbor joining (NJ) trees were then independently generated for these mitochondrial and chloroplast SNPs to identify the phylogenetic relationships among these seven melon cultivars and the control DHL92 (Fig. 4). We found that the mitochondrial tree (Fig. 4A) was well resolved and the branch support value for resolved nodes was over $70 \%$ bootstrap. The geographic location and three agronomic traits (fruit shape, fruit skin color, and seed type) of these seven melon cultivars were plotted on the phylogenetic tree to determine whether any of these melon characters corresponded to the relationships among these cultivars (Fig. 4A). Our data showed that the mitochondrial tree topology could not be used for identification of the melon features. However, the $\mathrm{MP} / \mathrm{NJ}$ tree of the chloroplast SNPs was well resolved or supported their defined melon features between phylogenet-

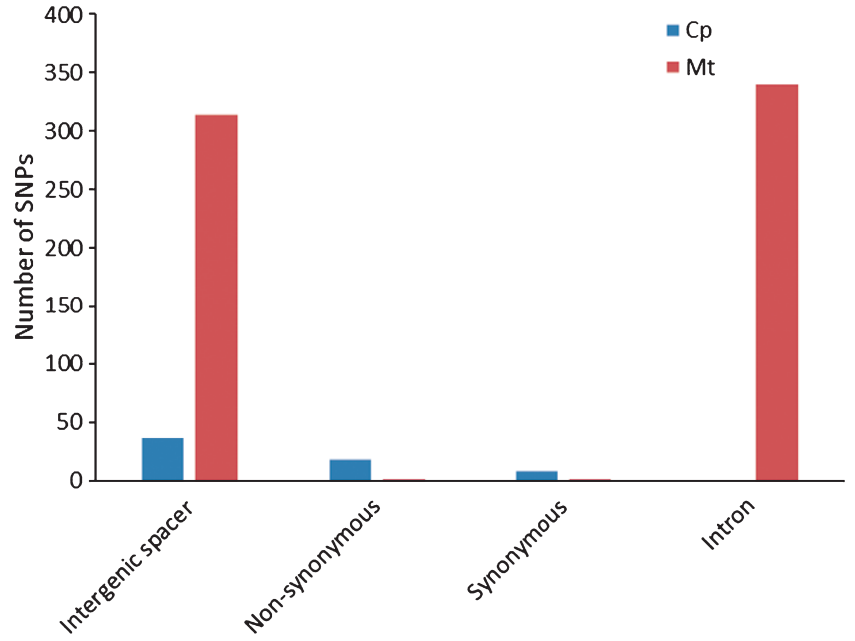

Fig. 3. Localization of the mitochondrial and chloroplast single nucleotide polymorphisms (SNPs). Data show their localization in intergenic spacers, introns, and protein coding genes. For the SNPs in coding regions, the number that results in synonymous versus nonsynonymous substitutions is indicated.

ic tree and the seed traits (Fig. 4B), including seed length, width, thickness, 100-seed weight, and type. The large-seed melons with seed length $\geq 9 \mathrm{~mm}$ and 100 -seed weight $\geq 2.5 \mathrm{~g}$ were divided into one clade, while the small-seed melons with seed length $<9 \mathrm{~mm}$ and 100 -seed weight $<2.5 \mathrm{~g}$ were classified in another clade. Moreover, the seed width and thickness in the large-seed melons (width $\geq 4.0 \mathrm{~mm}$ and thickness $\geq 1.5 \mathrm{~mm}$ ) were greater than those in the smallseed melons (width $\leq 3.3 \mathrm{~mm}$ and thickness $\leq 1.4 \mathrm{~mm}$ ). However, the bootstrap values of most of the nodes were slightly less than $50 \%$, which could be attributed to the fact that these 63 SNPs were either unique to a single cultivar or shared by all seven cultivars compared to the reference (Supplemental Table 1). The resulting MP/NJ tree topology of the nuclear genome was more resolved and better supported than both the genome-wide organellar phylogenies, in addition to a fine correspondence between the MP/ NJ tree topology and seed features (Fig. 4C).

\section{Discussion}

In this study, we assessed SNPs from the mitochondrial, chloroplast, and nuclear genomes to identify the phylogenetic associations among these seven melon cultivars after DNA sequencing of their genomes. We found that there were 207-295 mitochondrial SNPs, with a total of 658 SNPs, while there were 0-60 chloroplast SNPs among these seven melon cultivars compared to the reference genome. Of the mitochondrial SNPs, 182 SNPs were unique to a single cultivar and resulted from only three melon cultivars (Topmark, M4-5 and M1-15). Most of these SNPs were localized in the intergenic spacer and intron regions; only two nonsynonymous substitutions (SJF412792_222328 and SJF412792_222329) and two synonymous substitutions 

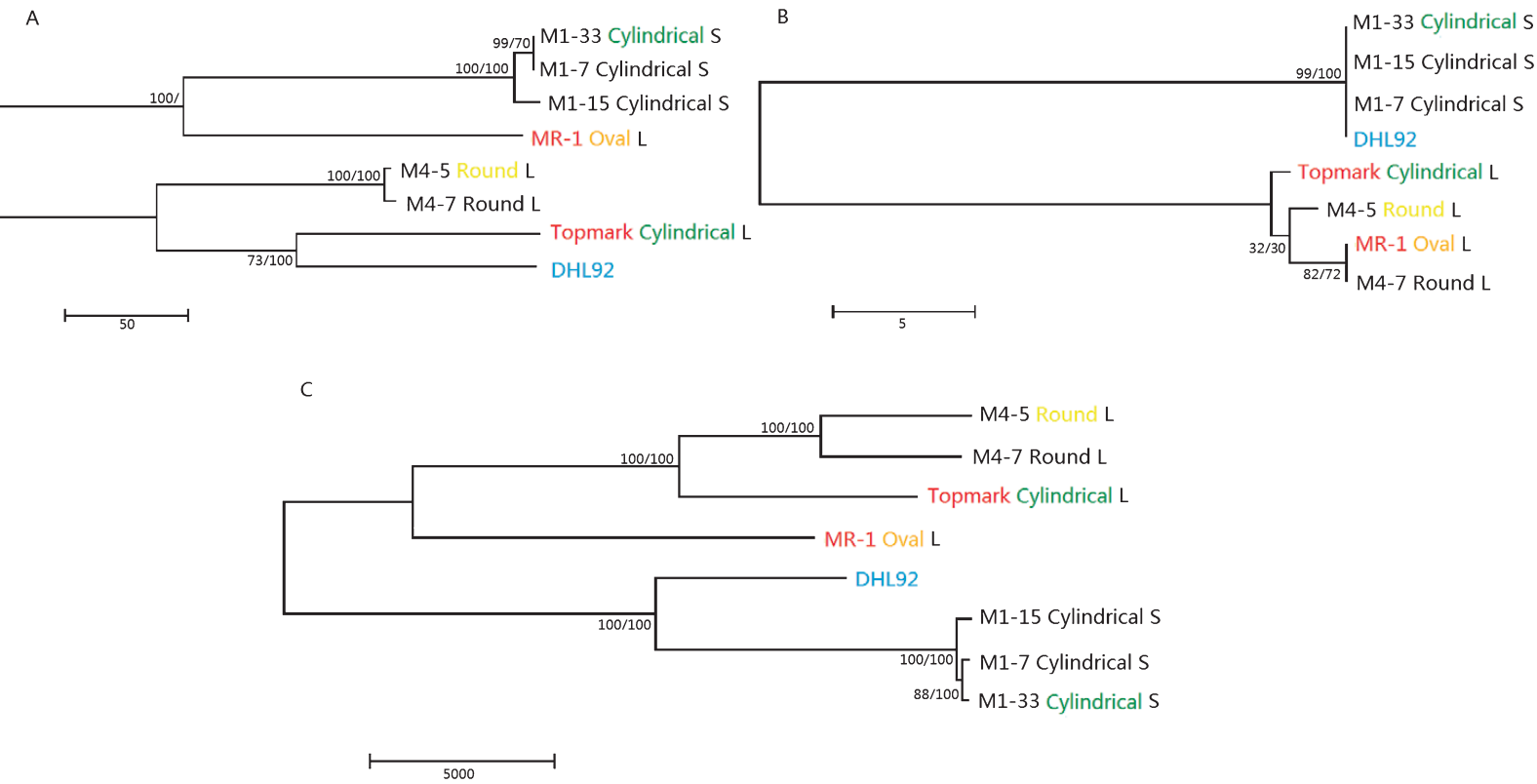

Fig. 4. Illustration of the best-fitting maximum parsimony (MP) tree and the relationships of seven melon cultivars and DHL92 based on mitochondrial (A), chloroplast (B), and nuclear (C) single nucleotide polymorphisms (SNPs). Branch support values represent the MP and neighborjoining (NJ)-derived bootstrap values (MP/NJ). Cultivar name in black, red, and blue indicates their origination from China, America, and Spain, respectively. Fruit shape is indicated after the cultivar name and colored by fruit skin color (black represents white skin color). The labels "L" and "S" after the melon fruit shape correspond to large-seed and small-seed type, respectively.

(SJF412792_2137706 and SJF412792_2138399) were localized in the rps 1 and atpl gene, respectively. In contrast, all the chloroplast SNPs was from the large-seed melons while none was observed among the small-seed melons or the reference chloroplast genome. Near half of these chloroplast SNPs were in the exons or the intergenic spacer region, but not in the intron regions. We found that the mitochondrial tree topology failed to distinguish melon features, whereas the MP/NJ tree of the chloroplast SNPs could define melon features such as seed length, width, thickness, 100 -seed weight, and type. The SNPs of the nuclear genome were better than the SNPs of the mitochondrial and chloroplast genomes. Our current data demonstrated that mitochondrial, chloroplast, and nuclear SNPs differ in their usefulness to identify phylogenetic relationships among these seven melon cultivars. Future studies to further investigate the functions of these SNPs with regard to mRNA transcription and protein coding are warranted.

The mitochondria and chloroplast were originally derived from independent living cyanobacteria and proteobacteria, respectively, and incorporated into plant cells during evolution (Zimorski et al. 2014). To date, up to 300 chloroplasts can be found in a single plant cell; chloroplasts have a significant role in photosynthesis (Boffey and Leech 1982). Most previous studies have focused on the nuclear genome, and the plastome and mitochondrial genome are generally considered as DNA "contamination" (Lutz et al. 2011), although a study on such DNA did historically yield some important targets for comparative plant studies (Straub et al. 2012). The transfer of plastid DNA to the mitochondrion is a common phenomenon, with $1-12 \%$ of published angiosperm mitochondrial genomes (Mower et al. 2012). In the melon, the mitochondrial genome contains a fraction (38.6 kb, 1.4\% of the total DNA sequences) of chloroplastlike DNA sequences (Rodriguez-Moreno et al. 2011), whereas watermelon and squash consist of approximately $6.4 \%(24 \mathrm{~kb})$ and $2.1 \%(21 \mathrm{~kb})$ of sequences, respectively (Alverson et al. 2010). Further, $35 \%(54 \mathrm{~kb})$ of the melon chloroplast genome showed homology to the mitochondrial genome, and $46.5 \%$ (1.14 Mb) of the melon mitochondrial genome seems to be of nuclear origin (Rodriguez-Moreno et al. 2011), making the analysis of mitochondrial and chloroplast SNPs difficult. However, our current study indicated that the mitochondrial and chloroplast SNPs are unique and are useful for identification of phylogenetic relationships and melon features, which is consistent with previous studies (Tanaka et al. 2013).

In the past, different melon cultivars have been usually distinguished by their size, color, and the shape of their fruit or seed. Although classification based on these factors does have its merits, this method is not reliable and the melons are often misclassified. Molecular biomarkers have been increasingly used to define or precisely identify cultivars. In our current study, we performed whole genome DNA sequencing of mitochondrial, chloroplast, and nuclear genomes to identify SNPs in order to analyze the phylogenetic relationships among these seven melon cultivars. We revealed the unique SNPs in five of these seven cultivars. To our knowledge, this study is the first to report intra-specific diversification based on the comprehensive comparative 


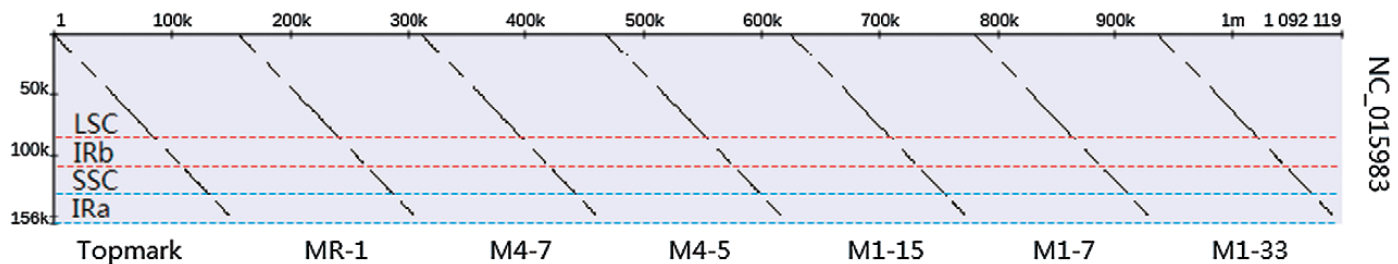

Fig. 5. Coverage of chloroplast genome of seven melon accessions after filtering.

analysis of the mitochondrial, chloroplast, and nuclear genomes of $C$. melo L. Previous studies have investigated chloroplast SNPs in melon (Sebastian et al. 2010, Tanaka et al. 2013), one of which showed that the SNPs of five chloroplast and two nuclear ribosomal DNA internal transcribed spacer (ITS) regions could identify the intra-specific diversification of the cytoplasm type within 17 melon accessions (Sebastian et al. 2010). Another study showed that chloroplast SNPs could also separate 60 melon accessions into three subclusters (Ia, Ib, or Ic) (Tanaka et al. 2013), and that the subclusters Ia and $\mathrm{Ib}$ mainly consist of small-seed and large-seed melons, respectively, while the subcluster Ic that occurred in both large- and small-seed melons was unique to Africa. The subcluster Ic can be characterized by eight chloroplast SNPs, while subclusters Ia and Ib can be characterized by seven SNPs (Tanaka et al. 2013). In our current study, we revealed that 658 and 63 SNPs in the inter- and intra-genic regions covered approximately $95 \%$ and $64 \%$ of the mitochondrial and chloroplast genome, respectively. These intercultivar SNPs of the mitochondrial and chloroplast genomes could be used to separate small- and largeseed melon cultivars. The number of SNPs in the mitochondrial and chloroplast genomes is small, facilitating their use. Caspermeyer (2015) and Sanseverino et al. (2015) reported over 4.3 million SNPs in the nuclear genome of the seven melon varieties.

Further, our current data on the SNPs of the chloroplast and nuclear genome are largely consistent with previous studies on melon accession and phylogenetic analysis (Tanaka et al. 2013). Our data on inconsistent phylogenies of these three genomes may provide a different insight into the complex evolutionary history of the melon. This is also the first study to directly compare chloroplast and mitochondrial phylogenetic reconstructions within C. melo. Despite the fact that only $64 \%$ data $(42 \%$ of the inverted repeats and $77 \%$ of the single-copy regions) on the chloroplast genome was used for chloroplast phylogenetic analysis (Fig. 5), our chloroplast phylogeny was well resolved, with high bootstrap support for major nodes. Our current data also showed that the nuclear phylogeny had compatibilities with both the mitochondrial and chloroplast phylogenies, but these two cytoplasmic phylogenies are not consistent with each other. Our chloroplast phylogeny is consistent with the hypothesis of Tanaka et al. (2013), which showed that the small-seed melon was placed in one clade, while the large-seed melon was included in another clade. In contrast, in the mitochondrial genome phylogeny, melon MR-1 cultivar has been placed into the clade opposite to chloroplast and the nuclear phylogenetic tree. Despite the limited amount of sequence variation in the cytoplasmic genome sequence, the incongruence was remarkable. Incongruence is expected when the mitochondrial and chloroplast genomes exhibit contrasting paternal and maternal modes of inheritance. Such discordance is common in the genus Cucumis, where the chloroplast is maternally inherited and the mitochondria are paternally inherited (Havey et al. 1998, Shen et al. 2013, Wei et al. 2011, Zhao et al. 2014). The mitochondrial phylogenetic tree could reflect MR-1 from a small-seed paternal ancestor, while the chloroplast phylogenetic tree could reveal that the large-seed melon had a maternal ancestor of MR-1. As is well known, MR-1 was selected from PI 124111 (Thomas 1986); the 100-seed weight of PI 124111 exceeds $2.5 \mathrm{~g}$. Thus, it is a large-seed type melon (https://npgsweb.ars-grin.gov). However, our current data showed that the genome-wide mitochondrial, chloroplast, and nuclear MP/NJ trees were not well consistent with fruit shape, fruit skin color or geographical location, although chloroplast and nuclear MP/NJ trees were consistent with the length, width, and thickness, and type of seed and 100-seed weight. Future studies that further assess SNPs to classify different melons worldwide are warranted.

\section{Acknowledgments}

This study was supported by a grant from the China Agriculture Research System (\#CARS-26-02).

\section{Literature Cited}

Allen, G.C., M.A. Flores-Vergara, S. Krasynanski, S. Kumar and W.F. Thompson (2006) A modified protocol for rapid DNA isolation from plant tissues using cetyltrimethylammonium bromide. Nat. Protoc. 1: 2320-2325.

Alverson, A.J., X. Wei, D.W. Rice, D.B. Stern, K. Barry and J.D. Palmer (2010) Insights into the evolution of mitochondrial genome size from complete sequences of Citrullus lanatus and Cucurbita pepo (Cucurbitaceae). Mol. Biol. Evol. 27: 1436-1448.

Boffey, S.A. and R.M.Leech (1982) Chloroplast DNA levels and the control of chloroplast division in light-grown wheat leaves. Plant physiol. 69: 1387-1391.

Boualem,A., M.Fergany, R.Fernandez, C.Troadec, A.Martin, H. Morin, M.A. Sari, F. Collin, J.M. Flowers, M. Pitrat et al. (2008) A conserved mutation in an ethylene biosynthesis enzyme leads to 
andromonoecy in melons. Science 321: 836-838.

Caspermeyer, J. (2015) Melon genome study reveals recent impacts of breeding. Mol. Biol. Evol. 32: 2801-2802.

Chung, S.M., J.E. Staub and J.F. Chen (2006) Molecular phylogeny of Cucumis species as revealed by consensus chloroplast SSR marker length and sequence variation. Genome 49: 219-229.

Cingolani, P., V.M.Patel, M.Coon, T.Nguyen, S.J.Land, D.M.Ruden and X.Lu (2012) Using drosophila melanogaster as a model for genotoxic chemical mutational studies with a new program, SnpSift. Front. Genet. 3: 35.

Gao, P., H.Ma, F.Luan and H.Song (2012) DNA fingerprinting of Chinese melon provides evidentiary support of seed quality appraisal. PLoS ONE 7: e52431.

Garcia-Mas, J., M.Oliver, H. Gómez-Paniagua and M.C. de Vicente (2000) Comparing AFLP, RAPD and RFLP markers for measuring genetic diversity in melon. Theor. Appl. Genet. 101: 860-864.

Garcia-Mas, J., A. Benjak, W. Sanseverino, M. Bourgeois, G. Mir, V.M. González, E. Hénaff, F. Câmara, L. Cozzuto, E. Lowy et al. (2012) The genome of melon (Cucumis melo L.). Proc. Natl. Acad. Sci. USA 109: 11872-11877.

Govindarajulu, R., M. Parks, J.A. Tennessen, A. Liston and T.L.Ashman (2015) Comparison of nuclear, plastid, and mitochondrial phylogenies and the origin of wild octoploid strawberry species. Am. J. Bot. 102: 544-554.

Harel-Beja, R., G.Tzuri, V.Portnoy, M.Lotan-Pompan, S.Lev, S. Cohen, N.Dai, L. Yeselson, A. Meir and S.E.Libhaber (2010) A genetic map of melon highly enriched with fruit quality QTLs and EST markers, including sugar and carotenoid metabolism genes. Theor. Appl. Genet. 121: 511-533.

Havey, M.J. (1997) Predominant paternal transmission of the mitochondrial genome in cucumber. J. Hered. 88: 232-235.

Havey, M.J., J.D. McCreight, B. Rhodes and G. Taurick (1998) Differential transmission of the Cucumis organellar genomes. Theor. Appl. Genet. 97: 122-128.

Ibdah, M., V.Portnoy, E.Bar, Y.Burger, A.A. Schaffer and Y.Tadmor (2006) Functional characterization of $\mathrm{CmCCD1}$, a carotenoid cleavage dioxygenase from melon. Phytochemistry 67: 15791589 .

Koboldt, D.C., Q.Zhang, D.E. Larson, D. Shen, M.D. McLellan, L.Lin, C.A. Miller, E.R. Mardis, L. Ding and R.K. Wilson (2012) VarScan 2: somatic mutation and copy number alteration discovery in cancer by exome sequencing. Genome Res. 22: 568-576.

Larkin, M.A., G. Blackshields, N.P. Brown, R. Chenna, P.A. McGettigan, H. McWilliam, F. Valentin, I.M. Wallace, A. Wilm, R. Lopez et al. (2007) Clustal W and Clustal X version 2.0. Bioinformatics 23: 2947-2948.

Li,H., B.Handsaker, A.Wysoker, T.Fennell, J.Ruan, N.Homer, G. Marth, G.Abecasis and R. Durbin (2009) The sequence alignment/ map format and SAMtools. Bioinformatics 25: 2078-2079.

Li,H. (2013) Aligning sequence reads, clone sequences and assembly contigs with BWA-MEM. Eprint Arxiv. ArXiv:1303.3997.

Lutz, K.A., W. Wang, A.Zdepski and T.P. Michael (2011) Isolation and analysis of high quality nuclear DNA with reduced organellar DNA for plant genome sequencing and resequencing. BMC Biotechnol. 11: 54

Martin,A., C. Troadec, A. Boualem, M. Rajab, R. Fernandez, H. Morin, M.Pitrat, C. Dogimont and A.Bendahmane (2009) A transposoninduced epigenetic change leads to sex determination in melon. Nature 461: 1135-1138.

Mower, J., D. Sloan and A.Alverson (2012) Plant mitochondrial genome diversity: the genomics revolution. In: Wendel, J.F., J.
Greilhuber, J. Dolezel and I.J.Leitch (eds.) Plant Genome Diversity, Springer, Vienna, pp. 123-144.

Nakata,E., J.E. Staub, A.I.López-Sesé and N.Katzir (2005) Genetic diversity of japanese melon cultivars (Cucumis melo L.) as assessed by random amplified polymorphic DNA and simple sequence repeat markers. Genet. Resour. Crop Evol. 52: 405-419.

Pech, J.C., M. Bouzayen and A. Latché (2008) Climacteric fruit ripening: Ethylene-dependent and independent regulation of ripening pathways in melon fruit. Plant Sci. 175: 114-120.

Renner, S.S., H. Schaefer and A.Kocyan (2007) Phylogenetics of Cucumis (Cucurbitaceae): cucumber (C. sativus) belongs in an Asian/Australian clade far from melon (C. melo). BMC Evol. Biol. 7: 58.

Rodriguez-Moreno,L., V.M.Gonzalez, A.Benjak, M.C.Marti, P. Puigdomenech, M.A.Aranda and J. Garcia-Mas (2011) Determination of the melon chloroplast and mitochondrial genome sequences reveals that the largest reported mitochondrial genome in plants contains a significant amount of DNA having a nuclear origin. BMC Genomics 12: 424

Sabir, J.S., D.Arasappan, A.Bahieldin, S.Abo-Aba, S.Bafeel, T.A. Zari, S.Edris, A.M.Shokry, N.O.Gadalla, A.M. Ramadan et al. (2014) Whole mitochondrial and plastid genome SNP analysis of nine date palm cultivars reveals plastid heteroplasmy and close phylogenetic relationships among cultivars. PLoS ONE 9: e94158.

Saladié,M., J.Cañizares, M.A.Phillips, M.Rodriguez-Concepcion, C.Larrigaudière, Y.Gibon, M. Stitt, J.E.Lunn and J.Garcia-Mas (2015) Comparative transcriptional profiling analysis of developing melon (Cucumis melo L.) fruit from climacteric and nonclimacteric varieties. BMC Genomics 16: 440.

Sanseverino, W., E. Henaff, C.Vives, S.Pinosio, W. Burgos-Paz, M. Morgante, S.E. Ramos-Onsins, J. Garcia-Mas and J.M. Casacuberta (2015) Transposon insertions, structural variations, and SNPs contribute to the evolution of the melon genome. Mol. Biol. Evol. 32: 2760-2774.

Sebastian, P., H. Schaefer, I.R. Telford and S.S. Renner (2010) Cucumber (Cucumis sativus) and melon (C. melo) have numerous wild relatives in Asia and Australia, and the sister species of melon is from Australia. Proc. Natl. Acad. Sci. USA 107: 14269-14273.

Shen, J., M.G.Kere and J.-F.Chen (2013) Mitochondrial genome is paternally inherited in Cucumis allotetraploid $(C . \times$ hytivus $)$ derived by interspecific hybridization. Sci. Hortic. 155: 39-42.

Straub, S.C., M.Parks, K. Weitemier, M.Fishbein, R.C.Cronn and A. Liston (2012) Navigating the tip of the genomic iceberg: Nextgeneration sequencing for plant systematics. Am. J. Bot. 99: 349364.

Swofford, D.L. (2002) PAUP*. Phylogenetic analysis using parsimony (*and other methods). Version 4.0b10. Sinauer Associates, Sunderland, MA, USA.

Tamura, K., G. Stecher, D.Peterson, A.Filipski and S. Kumar (2013) MEGA6: Molecular evolutionary genetics analysis version 6.0. Mol. Biol. Evol. 30: 2725-2729.

Tanaka, K., A.Nishitani, Y.Akashi, Y.Sakata, H.Nishida, H. Yoshino and K. Kato (2007) Molecular characterization of south and east asian melon, Cucumis melo L., and the origin of group Conomon var. makuwa and var. conomon revealed by RAPD analysis. Euphytica 153: 233-247.

Tanaka,K., Y.Akashi, K.Fukunaga, T.Yamamoto, Y.Aierken, H. Nishida, C.L.Long, H. Yoshino, Y. Sato and K. Kato (2013) Diversification and genetic differentiation of cultivated melon inferred from sequence polymorphism in the chloroplast genome. Breed. Sci. 63: 183-196. 
Thomas, C.E. (1986) Downy and powdery mildew resistant muskmelon breeding line MR-1. Hortic. Sci. 21: 329.

Tzuri, G., X.Zhou, N.Chayut, H.Yuan, V.Portnoy, A.Meir, U.Sa'ar, F. Baumkoler, M. Mazourek, E. Lewinsohn et al. (2015) A 'golden' SNP in CmOr governs the fruit flesh color of melon (Cucumis melo). Plant J. 82: 267-279.

Vaughan, J.G. and C.A. Geissler (2009) The new oxford book of food plants, 2nd ed. Oxford University Press, USA, p. 134.

Wei, Y., G. H. Zhao., H. T. Yang and J. Chen (2011) Inheritance analysis of mitochondrial(mt) DNA in the interspecific crossing of Genus
Cucumis. J. Plant Genet. Resour.12: 612-618.

Zhang, T., Y.Fang, X. Wang, X. Deng, X.Zhang, S. Hu and J. Yu (2012) The complete chloroplast and mitochondrial genome sequences of Boea hygrometrica: insights into the evolution of plant organellar genomes. PLoS ONE 7: e30531.

Zhao, J., J.Shen, H.Li, Q.Lou, J.Li and J.Chen (2014) Detection of the paternal mitochondrial inheritance in Cucumis. Acta Hortic. Sin. 41: 2250-2258.

Zimorski, V., C. Ku, W.F. Martin and S.B. Gould (2014) Endosymbiotic theory for organelle origins. Curr. Opin. Microbiol. 22: 38-48. 